\title{
Probing Quantum Speed Limits with Ultracold Gases
}

\author{
Adolfo del Campo \\ Department of Physics and Materials Science, University of Luxembourg, L-1511 Luxembourg, Luxembourg; \\ Donostia International Physics Center, E-20018 San Sebastián, Spain; \\ IKERBASQUE, Basque Foundation for Science, E-48013 Bilbao, Spain; \\ Department of Physics, University of Massachusetts, Boston, Massachusetts 02125, USA \\ and Theory Division, Los Alamos National Laboratory, MS-B213, Los Alamos, New Mexico 87545, USA
}

(Received 2 August 2020; accepted 31 March 2021; published 7 May 2021)

\begin{abstract}
Quantum speed limits (QSLs) rule the minimum time for a quantum state to evolve into a distinguishable state in an arbitrary physical process. These fundamental results constrain a notion of distance traveled by the quantum state, known as the Bures angle, in terms of the speed of evolution set by nonadiabatic energy fluctuations. I theoretically propose how to measure QSLs in an ultracold quantum gas confined in a timedependent harmonic trap. In this highly-dimensional system of continuous variables, quantum tomography is prohibited. Yet, QSLs can be probed whenever the dynamics is self-similar by measuring as a function of time the cloud size of the ultracold gas. This makes it possible to determine the Bures angle and energy fluctuations, as I discuss for various ultracold atomic systems.
\end{abstract}

DOI: 10.1103/PhysRevLett.126.180603

The time-energy uncertainty relation is a fundamental result in quantum physics relating characteristic times to the inverse of energy fluctuations [1,2]. This seminal result goes back to Mandelstam and Tamm, who established it rigorously in 1945 [3]. Its modern formulation relies on quantum speed limits (QSLs) that bound the minimum time for a physical process to unfold in terms of energy fluctuations. QSLs render quantum dynamics with a geometric interpretation in which the quantum state of a system evolves in time by sweeping a distance in Hilbert space [4]. Thus, QSLs involve the notions of speed and distance in Hilbert space. Quantifying the distance between the initial and time-evolving quantum states requires estimating state overlaps, which is challenging, if not unfeasible, for manyparticle systems with continuous variables. Different norms of the generator of evolution provide upper bounds to the speed at which this distance is traversed. Apart from the standard deviation of the energy [2,3,5-9], the mean energy above the ground state has been widely used after the QSL introduced by Margolus and Levitin [10,11]. In addition, other moments of the Hamiltonian can be used to upper bound the speed of evolution [12,13], and, in certain settings, other notions of speed based on work fluctuations have been shown to be dominant [14].

By now, QSLs are established in open quantum systems [15-18] and stochastic evolutions under continuous quantum measurements $[19,20]$. Indeed, it is at present understood that speed limits are not restricted to the quantum domain and can be formulated universally using the tools of information geometry [21]. The derivation of speed limits in classical dynamics and stochastic thermodynamics constitute a compelling advance to this end [22-24].
The notion of distinguishability in classical and quantum systems is, however, fundamentally different. In the quantum domain, the default notion relies on the Bures angle $[8,25]$. Alternatively, other measures such as the WignerYanase information [26] and the generalized Bloch angle [27] have been explored.

In spite of the fundamental nature of QSLs, there is currently a lack of experimental studies probing them. In this work, I propose the experimental study of QSLs with many-body systems of trapped ultracold atoms by measuring the mean atomic cloud size as a function of the evolution time. I show that, for scale-invariant many-body systems, the Mandelstam-Tamm QSL can be probed, given that the Bures angle as well as the nonadiabatic energy fluctuations can be determined from the mean atomic cloud size, which is an experimentally measurable quantity.

Geometry of quantum dynamics and QSL.-The degree to which two pure quantum states resemble each other is captured by the absolute square value of their overlap, i.e., their fidelity. Consider an initial quantum state $|\Psi(0)\rangle$ and its time evolution after a time $t$ denoted by $|\Psi(t)\rangle=U(t, 0)|\Psi(0)\rangle$, where $U(t, 0)$ is the unitary time-evolution operator generated by the system Hamiltonian dynamics assuming that the system is isolated from the external environment. The fidelity $F(t)=$ $|\langle\Psi(0)|U| \Psi(0)\rangle|^{2}$ gives the survival probability of the initial state after a time $t$ of evolution. A notion of distance between quantum states is provided by the Bures angle $[8,25]$. In particular, the Bures angle between the initial and the time-dependent states reads

$$
\mathcal{L}(t)=\mathcal{L}[|\Psi(0)\rangle, U|\Psi(0)\rangle]=\arccos \sqrt{F(t)} .
$$


The Bures angle swept during the evolution is upper bounded in terms of the quantum Fisher information $I_{Q}$ :

$$
\mathcal{L}(\tau) \leq \int_{0}^{\tau} d s \sqrt{I_{Q}(t) / 4} .
$$

Under unitary evolution, the quantum Fisher information is proportional to the energy variance, i.e.,

$$
I_{Q}(t)=\frac{4}{\hbar^{2}}\left[\left\langle\Psi(t)\left|H(t)^{2}\right| \Psi(t)\right\rangle-\langle\Psi(t)|H(t)| \Psi(t)\rangle^{2}\right] .
$$

This results in the Mandelstam-Tamm QSL [2,3,8,9]

$$
\tau \geq \tau_{\mathrm{QSL}}=\frac{\hbar \mathcal{L}(\tau)}{\overline{\Delta H}}
$$

where the mean energy dispersion reads

$$
\overline{\Delta H}=\frac{1}{\tau} \int_{0}^{\tau} d t \sqrt{\operatorname{var}_{\rho(t)}[H(t)]} .
$$

This QSL can be used to characterize a given evolution. To this end, I introduce the difference between the integrated nonadiabatic standard deviation of the energy and the Bures angle

$$
\delta \mathcal{L}(\tau)=\frac{1}{\hbar} \int_{0}^{\tau} d t \sqrt{\operatorname{var}_{\rho(t)}[H(t)]}-\mathcal{L}(\tau) \geq 0 .
$$

The first term in the rhs, $\gamma(\tau)=\tau \overline{\Delta H}$, represents the length of the path followed during the evolution in projective Hilbert space from $\Psi(0)$ to $\Psi(\tau)[7,28]$ :

$$
\gamma(\tau)=\int_{0}^{\tau} d t \sqrt{\left\langle d_{t} \Psi(t)|[1-P(t)]| d_{t} \Psi(t)\right\rangle}
$$

with $P(t)=|\Psi(t)\rangle\langle\Psi(t)|$. This length cannot be smaller than the actual geodesic $\mathcal{L}(\tau)$ between the two states, i.e., the distance defined by Eq. (1). Thus, the quantity $\delta \mathcal{L}(\tau)$ quantifies the extent to which a given evolution saturates the QSL. Said differently, when $\delta \mathcal{L}(\tau)$ vanishes, the evolution takes place at the maximum speed allowed by the Mandelstam-Tamm bound at all times during the considered time interval $[0, \tau]$.

Trapped ultracold gases with self-similar dynamics.-I next show how to determine the QSL in ultracold atomic gases. Consider the family of time-dependent Hamiltonians

$$
H(t)=\sum_{i=1}^{N}\left[\frac{\vec{p}_{i}^{2}}{2 m}+\frac{1}{2} m \omega(t)^{2} \vec{r}_{i}^{2}\right]+\sum_{i<j} V\left(\vec{r}_{i}-\vec{r}_{j}\right),
$$

describing $N$ particles in a harmonic trap. Particles interact with each other through a homogeneous pairwise potential fulfilling $V(\lambda \vec{r})=\lambda^{-2} V(\vec{r})$. Thanks to this scaling property, the dynamics is self-similar, i.e., scale invariant [29-31], a familiar feature in Bose-Einstein condensates [32,33]. An energy eigenstate $\Psi(0)$ of the Hamiltonian at $t=0$ with eigenvalue $E(0)$ evolves into

$$
\begin{aligned}
\Psi(t)= & \frac{1}{b^{\frac{D N}{2}}} \exp \left[i \frac{m \dot{b}}{2 \hbar b} \sum_{i=1}^{N} \vec{r}_{i}^{2}-i \int_{0}^{t} \frac{E(0)}{\hbar b\left(t^{\prime}\right)^{2}} d t^{\prime}\right] \\
& \times \Psi\left(\frac{\vec{r}_{1}}{b}, \ldots, \frac{\vec{r}_{N}}{b}, t=0\right),
\end{aligned}
$$

where $D$ denotes the spatial dimension and $b(t)$ is the scaling factor that determines the atomic cloud size. The specific time dependence of the latter following an arbitrary modulation of the trapping frequency $\omega(t)$ can be found by solving the Ermakov equation $\ddot{b}+\omega(t)^{2} b=\omega_{0}^{2} / b^{3}$, with the boundary conditions $b(0)=1$ and $\dot{b}(0)=0$, as $\Psi(0)$ is assumed to be stationary for $t<0$.

While the scale-invariant dynamics facilitates the description of the time evolution, the study of QSL remains hindered by the requirement to compute the Bures angle. Direct measurement of the overlap between quantum states is generally difficult in many-body systems, in particular, in the case of continuous variables. However, I shall show that, for a low-energy state in a variety of systems, the Bures angle can be expressed solely in terms of the scaling factor, which is an experimentally measurable quantity.

To relate the Bures angle to the ultracold-gas cloud size, first consider the system Hamiltonian in the absence of a trap:

$$
H_{\text {free }}=\sum_{i=1}^{N} \frac{\vec{p}_{i}^{2}}{2 m}+\sum_{i<j} V\left(\vec{r}_{i}-\vec{r}_{j}\right)
$$

and let $\psi_{\nu}$ be an energy eigenstate satisfying $H_{\text {free }} \psi_{\nu}=\varepsilon_{\nu} \psi_{\nu}$, that is also a homogeneous function:

$$
\psi_{\nu}\left(\lambda \vec{r}_{1}, \ldots, \lambda \vec{r}_{N}\right)=\lambda^{\nu} \psi_{\nu}\left(\vec{r}_{1}, \ldots, \vec{r}_{N}\right) ;
$$

i.e., it is an eigenstate of the dilatation operator $\sum_{i=1}^{N} \vec{r}_{i} \cdot \nabla_{\vec{r}_{i}} \psi_{\nu}=\nu \psi_{\nu}$. Then, the ground-state wave function of the Hamiltonian $H(0)=H_{\text {free }}+\frac{1}{2} m \omega_{0}^{2} \sum_{i=1}^{N} \vec{r}_{i}^{2}$ in Eq. (9) reads

$\Psi_{0}\left(\vec{r}_{1}, \ldots, \vec{r}_{N}\right)=c_{0} e^{-\left(m \omega_{0} / 2 \hbar\right) \sum_{i=1}^{N} \vec{r}_{i}^{2}} \psi_{\nu}\left(\vec{r}_{1}, \ldots, \vec{r}_{N}\right)$,

where $c_{0}$ is a normalization constant and the energy eigenvalue is $E(0)=\varepsilon_{\nu}+\hbar \omega_{0}[\nu+(D N / 2)]$. This relation between eigenstates in the presence and absence of a trap is realized in a variety of systems [34-37]. It holds (trivially) for the ground state of the single-particle harmonic oscillator. It also applies to the ground state of onedimensional many-body systems such as the free Bose gas, a polarized free Fermi gas, the Tonks-Girardeau gas and the Calogero-Sutherland gas [38]. In three spatial 
dimensions, it describes a family of states of the unitary Fermi gas $[39,40]$.

Upon varying $\omega(t)$, the self-similar evolution (8) yields

$\Psi_{0}(t)=\frac{c_{0} e^{i \alpha_{t}}}{b^{\nu+\frac{D N}{2}}} e^{-\left(m \omega_{0} / 2 \hbar\right)\left[\left(1 / b^{2}\right)-i\left(\dot{b} / \omega_{0} b\right)\right] \sum_{i=1}^{N} \vec{r}_{i}^{2}} \psi_{\nu}(0)$,

with $\alpha_{t}=-\int_{0}^{t}\left\{\left[E_{m}(0)\right] /\left[\hbar b\left(t^{\prime}\right)^{2}\right]\right\} d t^{\prime}$ being a dynamical phase. Thus, the overlap between $\Psi_{0}(0)$ and its time evolution at time $t$ equals

$\left\langle\Psi_{0}|U(t, 0)| \Psi_{0}\right\rangle=e^{i \alpha_{t}}\left[\frac{b}{2}\left(1+\frac{1}{b^{2}}-i \frac{\dot{b}}{\omega_{0} b}\right)\right]^{-\sigma^{2}}$,

where $\sigma^{2}=\nu+(D N / 2)$ in spatial dimension $D$.

Its absolute value is the square root of the fidelity used to define the Bures angle

$$
\sqrt{F(t)}=\left\{\frac{b^{2}}{4}\left[\left(1+\frac{1}{b^{2}}\right)^{2}+\left(\frac{\dot{b}}{\omega_{0} b}\right)^{2}\right]\right\}^{-\sigma^{2} / 2} \text {. }
$$

Further note that

$$
\sigma^{2}=\frac{E(0)}{\hbar \omega_{0}}=\frac{1}{x_{0}^{2}}\left\langle\Psi_{0}\left|\sum_{i=1}^{N} \vec{r}_{i}^{2}\right| \Psi_{0}\right\rangle
$$

which means that $\sigma$ is the initial size of the cloud formed by the ultracold gas in units of $x_{0}=\sqrt{\hbar /\left(m \omega_{0}\right)}$, that can be experimentally measured. As a result, the Bures angle swept during a time of evolution $t$ can be determined from the time-dependent scaling factor $b(t)$. Remarkably, the expression for the fidelity (14) holds for a variety of harmonically trapped quantum systems when $\left|\Psi_{0}\right\rangle$ is chosen to be the ground state with energy $E(0)$. See Ref. [34] for the derivation of the values of $\sigma^{2}$ summarized here: For a $D$-dimensional quantum oscillator $(N=1)$, $\sigma^{2}=D / 2$. For a trapped noninteracting Bose gas $(V=0)$, $\sigma^{2}=N D / 2$, while for a spin-polarized Fermi gas, $\sigma^{2}=N^{2} D / 2$. For bosonic systems in one spatial dimension $D=1$, whenever $V$ describes hard-core interactions, one recovers the Tonks-Girardeau gas [41,42], experimentally realized in Refs. [43-46]. In this case, $\sigma^{2}=N^{2} / 2$, which matches the result of a one-dimensional spin-polarized Fermi gas as a result of the Bose-Fermi mapping [47-49]. For the rational Calogero-Sutherland model in which $V$ represents inverse-square pairwise interactions of strength $\lambda$ [50-52], $\sigma^{2}=N[1+\lambda(N-1)] / 2$. In addition, for a unitary Fermi gas in three spatial dimensions [29,40, 53-55], one can make use of the general expression $\sigma^{2}=$ $E(0) /\left(\hbar \omega_{0}\right)[56]$.

The vanishing of the fidelity (14) for $t>0$ in many-body systems can be considered a manifestation of the orthogonality catastrophe [57], encoded in the dependence of $\sigma^{2}$ on the particle number $N$. In particular, the scaling $\sigma^{2} \propto N^{2}$ is shared not only by spin-polarized fermions and hard-core bosons [58,59], but as well by the CalogeroSutherland gas $[60,61]$.

Apart from the Bures angle, the study of QSL requires knowledge of the speed of evolution. Under scale-invariant dynamics generated by the time-dependent Hamiltonian (9), the energy variance in a state (8) is

$$
\operatorname{var}_{\rho(t)}[H(t)]=\hbar^{2} \omega(t)^{2} \sigma^{2}\left[\left(Q^{*}\right)^{2}-1\right] .
$$

Here, the nonadiabatic factor $Q^{*}(t)$ is given by

$$
Q^{*}(t)=\frac{\omega_{0}}{\omega(t)}\left[\frac{1}{2 b(t)^{2}}+\frac{\omega(t)^{2} b(t)^{2}}{2 \omega_{0}^{2}}+\frac{\dot{b}(t)^{2}}{2 \omega_{0}^{2}}\right]
$$

and accounts for the amount of energy excitations over the adiabatic dynamics. Indeed, $Q^{*}(t)=\langle H(t)\rangle /\langle H(t)\rangle_{\text {ad }}$ is the ratio between the nonadiabatic mean energy $\langle H(t)\rangle$ and the mean energy under adiabatic driving $\langle H(t)\rangle_{\mathrm{ad}}=$ $\langle H(0)\rangle \omega(t) / \omega_{0}$ [56,62-64]. Thus, the integrated mean energy dispersion is given by

$$
\gamma(\tau)=\sigma \int_{0}^{\tau} d t \sqrt{\omega(t)^{2}\left\{\left[Q(t)^{*}\right]^{2}-1\right\}},
$$

and, together with Eq. (14), it determines the QSL in Eq. (2). QSLs can, thus, be probed by determining $b(t)$ from time-of-flight imaging data, using a similar analysis to that used for determination of the nonadiabatic scaling factor $Q^{*}$ reported in Refs. [34,55].

Generic expansion versus a shortcut.-I next analyze the nonadiabatic expansion resulting from varying the trap frequency from an initial value $\omega_{0}$ to a final one $\omega_{F}<\omega_{0}$ in an expansion time $\tau$. I first consider a linear ramp $\omega(t)=\omega_{0}+\left(\omega_{F}-\omega_{0}\right) t / \tau$, for which the scaling factor $b(t)$ is determined by solving numerically the Ermakov equation. I compare it with a shortcut to adiabaticity (STA) designed by reverse engineering the scale-invariant dynamics $[31,34,65]$. The latter is based on fixing first a trajectory of the scaling factor $b(t)$ interpolating between the boundary conditions $b(0)=1$ and $b(\tau)=\sqrt{\omega_{0} / \omega_{F}}$, the latter being the target adiabatic value obtained by setting $\ddot{b} \approx 0$ in the Ermakov equation. For the initial and final states to be nonstationary, Eq. (8) imposes that $\dot{b}(0)=\dot{b}(\tau)=0$. The polynomial ansatz $b(t)=1+10(t / \tau)^{3}[b(\tau)-1]-$ $15(t / \tau)^{4}[b(\tau)-1]+6(t / \tau)^{5}[b(\tau)-1]$ is thus chosen, satisfying as well $\ddot{b}(0)=\ddot{b}(\tau)=0$, and the trap frequency $\omega(t)$ is determined from the Ermakov equation as $\omega(t)^{2}=\omega_{0} / b^{4}-\ddot{b} / b$.

Figure 1 shows the scaling factor as a function of time in a fast expansion. In the linear ramp, $b(t)$ does not reach the adiabatic value corresponding to the final frequency, $b(\tau)=\sqrt{\omega_{0} / \omega_{F}}=4$, while for the STA it interpolates for any $\tau$ between the initial and target configurations. 
(a)
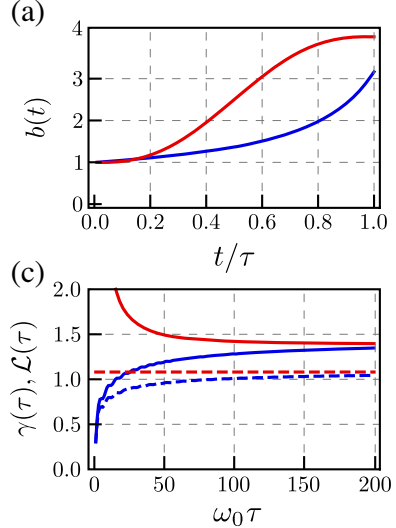

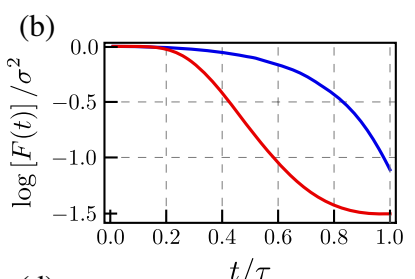

(d)

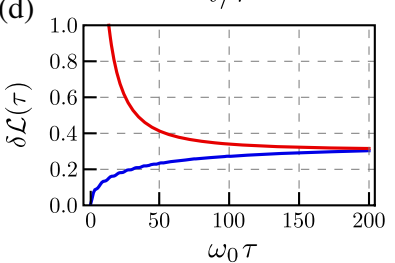

FIG. 1. QSL for an expansion induced by a linear frequency ramp and a shortcut to adiabaticity. (a) Scaling factor and (b) logarithmic fidelity as a function of time for a fourfold expansion with $\tau=10 / \omega_{0}$ for a linear ramp (blue line) and a STA (red line). Orthogonality catastrophe is encoded in the constant $\sigma^{2}$, which captures the dependence on the system size. (c) Path length $\gamma(\tau)$ (solid line) in Hilbert space lower bounded by the geodesic $\mathcal{L}(\tau)$ (dashed line) with $N=1$. (d) While the excess Bures angle $\delta \mathcal{L}$ increases for a linear ramp as the adiabatic limit is approached, the converse is true for the STA.

Given the scaling factor $b(t)$, I obtain the fidelity along the process using Eq. (14) and show its monotonic decay in both cases. Knowledge of the scaling factor also allows one to determine the integrated energy dispersion that sets the length $\gamma(\tau)$ of the path travel in Hilbert state and which is lower bounded by the geodesic $\mathcal{L}$. This demonstrates that the QSL is fulfilled during the dynamics, in any process. Moreover, the difference between $\gamma(\tau)$ and $\mathcal{L}(\tau)$ shows the extent to which the evolution saturates the QSL. For an arbitrary expansion time $\tau$, a linear ramp follows more closely the QSL than the STA but yields lower values of $\gamma(\tau)$ and $\mathcal{L}(\tau)$. Indeed, for fast expansions, both quantities vanish with a linear ramp, while a STA involves large deviations from QSL and has a $\mathcal{L}(\tau)$ independent of $\tau$. For slow expansions with $\omega_{0} \tau \gg 1$, both protocols behave alike. In the adiabatic limit, $\delta \mathcal{L}$ is still finite, as I next show.

Example 2. Adiabatic and transitionless quantum driving.-Counterdiabatic or transitionless quantum driving (TQD) is a technique that enforces the evolution of the state along a prescribed adiabatic trajectory [66-68]. To this end, an auxiliary control field is introduced to assist the dynamics and enforce parallel transport. Takahashi has shown that TQD solves the quantum brachistochrone [69], that is, the variational problem of minimizing the evolution time between an initial and a final state under fixed energy variance [70]. I analyze to what extent the resulting evolution minimizes $\delta \mathcal{L}(\tau)$.

For the time-dependent Hamiltonian (9), the adiabatic evolution can be obtained from Eq. (8) by considering the adiabatic scaling factor $b(t)=\sqrt{\omega_{0} / \omega(t)}$. Using this expression in Eq. (8) while setting $\dot{b} \approx 0$ yields

$$
\Psi(t)=\frac{e^{i \alpha_{t}}}{b^{D N / 2}} \Psi\left(\frac{\vec{r}_{1}}{b}, \ldots, \frac{\vec{r}_{N}}{b}, t=0\right) .
$$

The auxiliary control field that assists the dynamics along this adiabatic trajectory is given by $[71,72]$

$$
H_{1}(t)=\frac{\dot{b}}{b} C=\frac{\dot{b}}{b} \frac{1}{2} \sum_{i=1}^{N}\left\{\vec{r}_{i}, \vec{p}_{i}\right\},
$$

where $C$ is the squeezing operator. Thus, the evolution (19) is the exact solution of the many-body timedependent Schrödinger equation with the Hamiltonian $H_{T}=H(t)+H_{1}(t)$. In this case, the energy variance reduces to the second moment of the auxiliary term $\Delta H_{T}^{2}=$ $\left\langle H_{1}^{2}\right\rangle[73,74]$. The nonadiabatic energy variance can then be written as [34]

$$
\Delta H_{T}^{2}=\left(\frac{\dot{b}}{b}\right)^{2}\left\langle C^{2}(t)\right\rangle=\left(\frac{\dot{b}}{b}\right)^{2} \hbar^{2} \sigma^{2} .
$$

The Mandelstam-Tamm upper bound to the speed of evolution is, thus, governed by the second moment of the squeezing operator, which is time independent. Explicit integration yields the path length traveled $\gamma(\tau)$ :

$\int_{0}^{\tau} d t \frac{\Delta H_{T}(t)}{\hbar}=\sigma \alpha \log b(\tau)=\log \left(\frac{\omega(\tau)}{\omega_{0}}\right)^{-\alpha \sigma / 2}$,

assuming $b(t)$, and thus $\omega(t)$, to be monotonic. In this case, $\alpha=\operatorname{sgn}(\dot{b})$ reduces to +1 in an expansion and to -1 in a compression. Under TQD, the Bures angle is set by the overlap between the initial eigenstate of $H(0)$ and its adiabatic continuation (19) at time $t$ :

$$
F(\tau)=\left[\frac{\omega_{0}}{4 \omega(\tau)}\left(1+\frac{\omega(\tau)}{\omega_{0}}\right)^{2}\right]^{-\sigma^{2}} .
$$

In this case, the excess Bures angle reads

$$
\delta \mathcal{L}(\tau)=-\alpha \frac{\sigma}{2} \log x-\arccos \left[\left(\frac{1+x}{2 \sqrt{x}}\right)^{-\sigma^{2}}\right],
$$

where $x=\omega(\tau) / \omega_{0}$, and is shown in Fig. 2. For small expansions and compressions,

$$
\delta \mathcal{L}(\tau)=\alpha \sigma(1-x)+\frac{\alpha \sigma}{2}(1-x)^{2}+\mathcal{O}\left[(x-1)^{3}\right] .
$$

As a result, the excess Bures angle $\delta \mathcal{L}(\tau)$ remains finite for any ratio between the final and the initial frequency $x=\omega(\tau) / \omega_{0} \neq 1$. The characteristic range of the frequency ratio in which $\delta \mathcal{L}(\tau)$ is negligible is set by the inverse of $\sigma$. It is, thus, reduced for many particle systems as the particle number $N$ and the spatial dimension $D$ are increased. The results (22)-(25) not only describe TQD but 


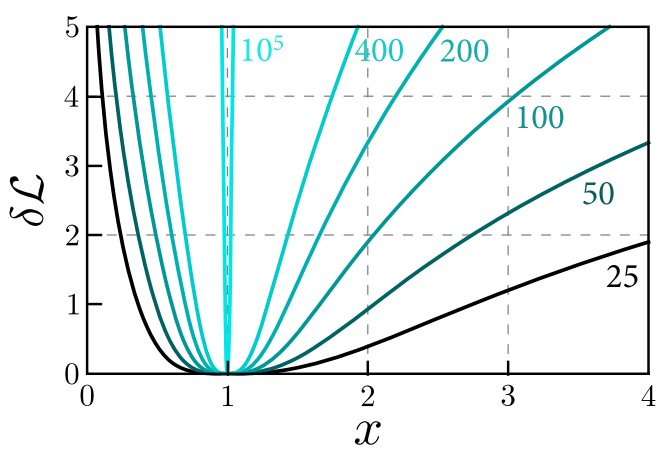

FIG. 2. Excess Bures angle under adiabatic evolution and TQD. $\delta \mathcal{L}(\tau)$ is shown for different values of $\sigma^{2}$, increasing from bottom to top. The Mandelstam-Tamm QSL is saturated only when the ratio $x=\omega(\tau) / \omega_{0}$ approaches unity, as $\delta \mathcal{L}(\tau)$ vanishes. Many particle effects increase $\delta \mathcal{L}(\tau)$, hindering the driving at the QSL.

also apply in the adiabatic limit [34]. Indeed, Eq. (24) for $x=1 / 16$ yields $\delta \mathcal{L}(\tau) \approx 0.305$, the asymptotic value in Fig. 1 for large $\tau$. In short, the QSL defined in terms of the Bures angle is never saturated, as the geodesic $\mathcal{L}(\tau)$ cannot be accessed with the considered dynamics. The reachable geodesic for $\gamma(\tau)$ is the one defined on the manifold of adiabatically accessible states, as conjectured in Ref. [74] for arbitrary dynamics.

Summary and conclusions. - I have demonstrated that QSLs can be probed in ultracold atom experiments characterized by self-similar dynamics. This proposal relies on measuring the size of the atomic cloud in a given process, such an expansion or compression driven by a modulation of the trap frequency. The scaling factor can be determined by imaging the cloud size via standard time-of-flight measurements or nondestructive Faraday imaging [75], among other approaches. From it, one can determine the distance traveled by the quantum state of the system in Hilbert space (Bures angle) during the evolution. This approach circumvents the need for quantum state tomography of the many-body state of a continuous variable system. In addition, the scaling factor also determines the Mandelstam-Tamm quantum speed of evolution, that equals the time average of the energy dispersion. Their knowledge allows one to quantify the extent to which a given evolution saturates the QSL, paving the way to identifying time-optimal protocols [76], as I have discussed in the context of fast control by shortcuts to adiabaticity. This proposal is amenable to experimental studies with trapped ultracold atomic clouds in three spatial dimensions (e.g., Ref. [77]). Similarly, it can be applied to ultracold gases in tight waveguides with an axial harmonic confinement [49]. In an isotropic setting, it can further be implemented at strong coupling using a unitary Fermi gas [53]. These results pave the way to the experimental study of the time-energy uncertainty relation and QSLs in many-body quantum systems and their relation to the orthogonality catastrophe.
It is a pleasure to acknowledge discussions with Andrea Alberti, Tommaso Calarco, Léonce Dupays, Íñigo L. Egusquiza, Fernando J. Gómez-Ruiz, Dries Sels, Kazutaka Takahashi, and Zhenyu Xu. This work was supported by the Spanish Ministry of Science and Innovation (PID2019-109007GA-I00).

[1] P. Pfeifer and J. Fröhlich, Rev. Mod. Phys. 67, 759 (1995).

[2] P. Busch, The time-energy uncertainty relation, in Time in Quantum Mechanics, edited by J. Muga, R. S. Mayato, and Í. Egusquiza (Springer, Berlin, 2008), pp. 73-105.

[3] L. Mandelstam and I. Tamm, J. Phys. USSR 9, 249 (1945).

[4] S. Deffner and S. Campbell, J. Phys. A 50, 453001 (2017).

[5] A. Messiah, Quantum Mechanics, Vol. 1 (North-Holland, Amsterdam, 1961).

[6] K. Bhattacharyya, J. Phys. A 16, 2993 (1983).

[7] J. Anandan and Y. Aharonov, Phys. Rev. Lett. 65, 1697 (1990).

[8] A. Uhlmann, Phys. Lett. 161A, 329 (1992).

[9] P. Pfeifer, Phys. Rev. Lett. 70, 3365 (1993).

[10] N. Margolus and L. B. Levitin, Physica (Amsterdam) 120D, 188 (1998).

[11] V. Giovannetti, S. Lloyd, and L. Maccone, Nat. Photonics 5, 222 (2011).

[12] B. Zieliński and M. Zych, Phys. Rev. A 74, 034301 (2006).

[13] N. Margolus, arXiv:1109.4994.

[14] K. Funo, J.-N. Zhang, C. Chatou, K. Kim, M. Ueda, and A. del Campo, Phys. Rev. Lett. 118, 100602 (2017).

[15] M. M. Taddei, B. M. Escher, L. Davidovich, and R. L. de Matos Filho, Phys. Rev. Lett. 110, 050402 (2013).

[16] A. del Campo, I. L. Egusquiza, M. B. Plenio, and S. F. Huelga, Phys. Rev. Lett. 110, 050403 (2013).

[17] S. Deffner and E. Lutz, Phys. Rev. Lett. 111, 010402 (2013).

[18] F. Campaioli, F. A. Pollock, and K. Modi, Quantum 3, 168 (2019).

[19] L. P. García-Pintos and A. del Campo, New J. Phys. 21, 033012 (2019).

[20] L. P. García-Pintos and A. del Campo, arXiv:1907.12574.

[21] S.-I. Amari, Information Geometry and Its Applications, Vol. AMS 194 (Springer, New York, 2016).

[22] B. Shanahan, A. Chenu, N. Margolus, and A. del Campo, Phys. Rev. Lett. 120, 070401 (2018).

[23] M. Okuyama and M. Ohzeki, Phys. Rev. Lett. 120, 070402 (2018).

[24] N. Shiraishi, K. Funo, and K. Saito, Phys. Rev. Lett. 121, 070601 (2018).

[25] W. K. Wootters, Phys. Rev. D 23, 357 (1981).

[26] D. P. Pires, M. Cianciaruso, L. C. Céleri, G. Adesso, and D. O. Soares-Pinto, Phys. Rev. X 6, 021031 (2016).

[27] F. Campaioli, F. A. Pollock, F. C. Binder, and K. Modi, Phys. Rev. Lett. 120, 060409 (2018).

[28] O. Andersson and H. Heydari, J. Phys. A 47, 215301 (2014).

[29] Y. Castin, C.R. Phys. 5, 407 (2004).

[30] V. Gritsev, P. Barmettler, and E. Demler, New J. Phys. 12, 113005 (2010).

[31] A. del Campo, Phys. Rev. A 84, 031606(R) (2011). 
[32] Y. Kagan, E. L. Surkov, and G. V. Shlyapnikov, Phys. Rev. A 54, R1753 (1996).

[33] Y. Castin and R. Dum, Phys. Rev. Lett. 77, 5315 (1996).

[34] See Supplemental Material at http://link.aps.org/supplemental/ 10.1103/PhysRevLett.126.180603 for more details.

[35] M. Olshanii, Phys. Rev. Lett. 81, 938 (1998).

[36] F. Werner and Y. Castin, Phys. Rev. A 74, 053604 (2006).

[37] M. Beau, J. Jaramillo, and A. del Campo, Entropy 18, 168 (2016).

[38] A. del Campo, Phys. Rev. Research 2, 043114 (2020).

[39] S. Tan, arXiv:cond-mat/0412764.

[40] Y. Castin and F. Werner, The unitary gas and its symmetry properties, in The BCS-BEC Crossover and the Unitary Fermi Gas, edited by W. Zwerger (Springer, Berlin, 2012), pp. 127-191.

[41] M. D. Girardeau, E. M. Wright, and J. M. Triscari, Phys. Rev. A 63, 033601 (2001).

[42] A. Minguzzi and D. M. Gangardt, Phys. Rev. Lett. 94, 240404 (2005).

[43] T. Kinoshita, T. Wenger, and D. S. Weiss, Science 305, 1125 (2004).

[44] B. Paredes, A. Widera, V. Murg, O. Mandel, S. Fölling, I. Cirac, G. V. Shlyapnikov, T. W. Hänsch, and I. Bloch, Nature (London) 429, 277 (2004).

[45] S. Palzer, C. Zipkes, C. Sias, and M. Köhl, Phys. Rev. Lett. 103, 150601 (2009).

[46] T. Jacqmin, J. Armijo, T. Berrada, K. V. Kheruntsyan, and I. Bouchoule, Phys. Rev. Lett. 106, 230405 (2011).

[47] M. Girardeau, J. Math. Phys. (N.Y.) 1, 516 (1960).

[48] G. Zürn, F. Serwane, T. Lompe, A. N. Wenz, M. G. Ries, J. E. Bohn, and S. Jochim, Phys. Rev. Lett. 108, 075303 (2012).

[49] A. N. Wenz, G. Zürn, S. Murmann, I. Brouzos, T. Lompe, and S. Jochim, Science 342, 457 (2013).

[50] F. Calogero, J. Math. Phys. (N.Y.) 12, 419 (1971).

[51] B. Sutherland, J. Math. Phys. (N.Y.) 12, 246 (1971).

[52] B. Sutherland, Phys. Rev. Lett. 80, 3678 (1998).

[53] S. Deng, Z.-Y. Shi, P. Diao, Q. Yu, H. Zhai, R. Qi, and H. Wu, Science 353, 371 (2016).

[54] S. Deng, P. Diao, Q. Yu, A. del Campo, and H. Wu, Phys. Rev. A 97, 013628 (2018).
[55] S. Deng, A. Chenu, P. Diao, F. Li, S. Yu, I. Coulamy, A. del Campo, and H. Wu, Sci. Adv. 4, eaar5909 (2018).

[56] M. Beau and A. del Campo, Entropy 22, 515 (2020).

[57] P. W. Anderson, Phys. Rev. Lett. 18, 1049 (1967).

[58] A. del Campo, Phys. Rev. A 84, 012113 (2011).

[59] T. Fogarty, S. Deffner, T. Busch, and S. Campbell, Phys. Rev. Lett. 124, 110601 (2020).

[60] A. del Campo, New J. Phys. 18, 015014 (2016).

[61] F. Ares, K. S. Gupta, and A. R. de Queiroz, Phys. Rev. E 97, 022133 (2018).

[62] J. Jaramillo, M. Beau, and A. del Campo, New J. Phys. 18, 075019 (2016).

[63] Obinna Abah and Eric Lutz, Europhys. Lett. 118, 40005 (2017).

[64] O. Abah and E. Lutz, Phys. Rev. E 98, 032121 (2018).

[65] X. Chen, A. Ruschhaupt, S. Schmidt, A. del Campo, D. Guéry-Odelin, and J. G. Muga, Phys. Rev. Lett. 104, 063002 (2010).

[66] M. Demirplak and S. A. Rice, J. Phys. Chem. A 107, 9937 (2003).

[67] M. Demirplak and S. A. Rice, J. Phys. Chem. B 109, 6838 (2005).

[68] M. V. Berry, J. Phys. A 42, 365303 (2009).

[69] K. Takahashi, J. Phys. A 46, 315304 (2013).

[70] A. Carlini, A. Hosoya, T. Koike, and Y. Okudaira, Phys. Rev. Lett. 96, 060503 (2006).

[71] C. Jarzynski, Phys. Rev. A 88, 040101(R) (2013).

[72] A. del Campo, Phys. Rev. Lett. 111, 100502 (2013).

[73] A. del Campo, M. M. Rams, and W. H. Zurek, Phys. Rev. Lett. 109, 115703 (2012).

[74] M. Bukov, D. Sels, and A. Polkovnikov, Phys. Rev. X 9 , 011034 (2019).

[75] M. Gajdacz, P. L. Pedersen, T. Mørch, A. J. Hilliard, J. Arlt, and J. F. Sherson, Rev. Sci. Instrum. 84, 083105 (2013).

[76] T. Caneva, M. Murphy, T. Calarco, R. Fazio, S. Montangero, V. Giovannetti, and G. E. Santoro, Phys. Rev. Lett. 103, 240501 (2009).

[77] J.-F. Schaff, X.-L. Song, P. Vignolo, and G. Labeyrie, Phys. Rev. A 82, 033430 (2010). 